\title{
Multiscale experimental and modelling framework for metal additive manufacturing : Hip implant case study
}

\section{Original Article}

\author{
Youssef Mohamed ${ }^{1}$, Khaled Nabil ${ }^{1}$, Nermeen Alaa ${ }^{1}$, Moustafa M. Abdel-Hamid ${ }^{2}$, \\ Mahmoud A. El-Sadek ${ }^{1}$, Khaled H. Khafagy ${ }^{1}$, Tarek M. Hatem ${ }^{1,3}$ \\ ${ }^{1}$ Center for Simulation Innovation and Advanced Manufacturing, the British University \\ in Egypt, Cairo, ${ }^{2}$ Mechanical Engineering Department, Nile University, Giza, ${ }^{3}$ Faculty \\ of Energy and Environmental Engineering, The British University in Egypt, Cairo, \\ Egypt.
}

\section{Keywords:}

Additive manufacturing, hip implant, metal, modelling framework, multiscale.

\section{Corresponding Author:}

Tarek M. Hatem, Faculty of Energy and Environmental Engineering, The British University in Egypt, Cairo, Egypt, Tel: +201069800019

Email:tarek.hatem@bue.edu.eg

\section{Abstract}

The current work targets improving metal-based Additive Manufacturing (AM) processes ; namely, Direct Metal Laser Sintering (DMLS). This research focuses on the manufacturing of steel alloys using the DMLS process, and high-strength 174- PH Stainless steel specifically. To achieve optimal material integrity and outstanding functionalities, different processing parameters impact on AM process are explored. Furthermore, development of a design framework based on general multi-scale models is suggested. The proposed framework includes: (1) Coupled thermal/microstructural prediction of DMLS process, (2) Establishing microstructure informed numerical models for properties and failure prediction for DMLS manufactured microstructures, (3) macroscale models with homogenized properties to be used for AM parts.

\section{INTRODUCTION}

Additive Manufacturing (AM) is an emerging and promising technique of manufacturing in high value-scale productivity. AM has a vast use in several industries such as aerospace, automotive, biomedical application, etc. Metal AM has many great advantages over traditional techniques such as reduced waste and faster in case of complex parts that requires many production stages or steps ${ }^{[1-2]}$.Therefore, as many industries and several applications require immediateproducts, they tend to use such technology for profit acceleration and better products accuracy. This research focuses on high-strength stainless steel, especially 17-4 PH stainless steel as a very had, stiff, and corrosion resistance material ${ }^{[3-4]}$.

To achieve optimal material integrity and outstanding functionalities, different processing parameters impact on AM process are explored. Furthermore, development of a design framework based on general multi-physics modelling is suggested. In this work, starting with a thermodynamics modelling to predict an accurate materials behaviours based on experimental testing, the materials properties can be predicated. The most important aspect in studying this manufacturing process is modelling the process itself. Materials Modelling is a great tool that improves our understanding about this process as the advancing materials modelling in piezo-electric smart materials ${ }^{[5-6]}$, and semiconductors ${ }^{[7-8]}$. Therefore, modelling the material by using the proposed multiscale materials modelling framework is crucial. Starting with microscale materials modelling using crystal plasticity based models targeting a macroscale modelling using an accurate numerical models along with finite element methods is the framework core. Furthermore, the numerical results are validated with the experimental results.

\section{METHODOLOGY}

AM processes are composed of complex multiphysical phenomena that include defects formation, phase transformation, and cyclic melting/solidification during the build process. These phenomenaincreased the finished part quality. For further advancements and understanding for AM materials behaviours, using multiscale modelling (see figure 1) of such materials (starting from nanoscale, microscale, to the macroscale) is crucial. As well as, these simulations directly improve and optimize the manufacturing process and fabrications for AM materials. Therefore, it will contribute to the final part quality and performance ${ }^{[9-12]}$. 


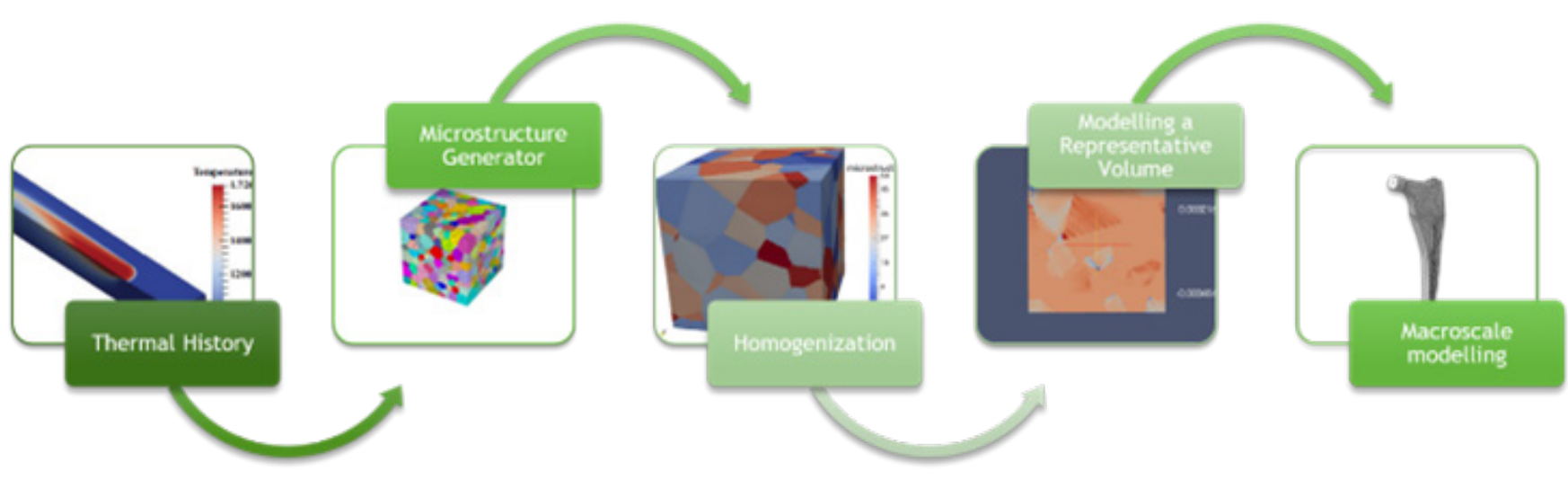

Fig. 1: Illustrative figure to show the multiscale modelling framework of Metal Additive Manufacturing.

The Calculation of Phase Diagrams (CALPHAD) approach a powerful engineering method in predicting material thermodynamics and kinetics of materials based on experimental phase equilibria and thermodynamic properties $^{[13]}$. In this manuscript, Scheil-Gulliver model is used along with the CALPHAD approach for phase equilibria, phase stability, and thermodynamic properties prediction and for the diffusion kinetic simulations and non-equilibrium studies during the solidification process.

At the macro scale, the thermal history is related to the mesoscale. The solution at the mesoscale gives information regarding the evolved microstructure properties; which can be passed up and homogenized to the macroscale to give an effective conductivity and phase composition.

Experimental studies will be developed to verify and validate the in-house process model implementation on its ability to predict thermal history and final microstructure within AM processes. Given a set of process parameters combined with a selected component geometry, the thermal/microstructure modelling technique will be used to predict the microstructure such as phases, porosity and grain size.

\section{RESULTS}

Thermodynamics modelling for AM process is critical where materials properties can be predicted. Such properties can be used in the multiscale materials modelling starting from the nanoscale reaching to the macroscale (product scale). Also, using accurate numerical materials models is crucial for better understanding of the materials behaviours and failure mechanisms ${ }^{[14-18]}$. Therefore, the following framework multiphysical models are presented;

\section{A- Thermodynamics Modelling}

Thermo-Calc ${ }^{\circledR}$, software will be used as it is the most popular and used software for this approach it has many advantages like ease of use, great functionality and unique features. Thermo-Calc $\AA$ can obtain:

- Calculating stable and meta-stable heterogeneous phase.

- Amount and composition of phases.

- Transformation temperatures, e.g. liquidus and solidus temperature.

- Predicting driving forces for phase transformations.

- Phase diagrams (binary, ternary, isothermal, isoplethal, etc.).

- Molar volume, density and thermal expansion.

- Scheil-Gulliver (non-equilibrium) solidification simulations.

- Thermochemical data such as:

o Enthalpies

o Heat capacity,

o Activities, etc.

- Thermodynamic properties of chemical reactions.

Figure 2 shows the property diagram of $\mathrm{Fe}-10 \mathrm{Ni}-0.1 \mathrm{C}$ as well as shows the number of phases exhibited in the material over a pre-assigned range of temperatures. 


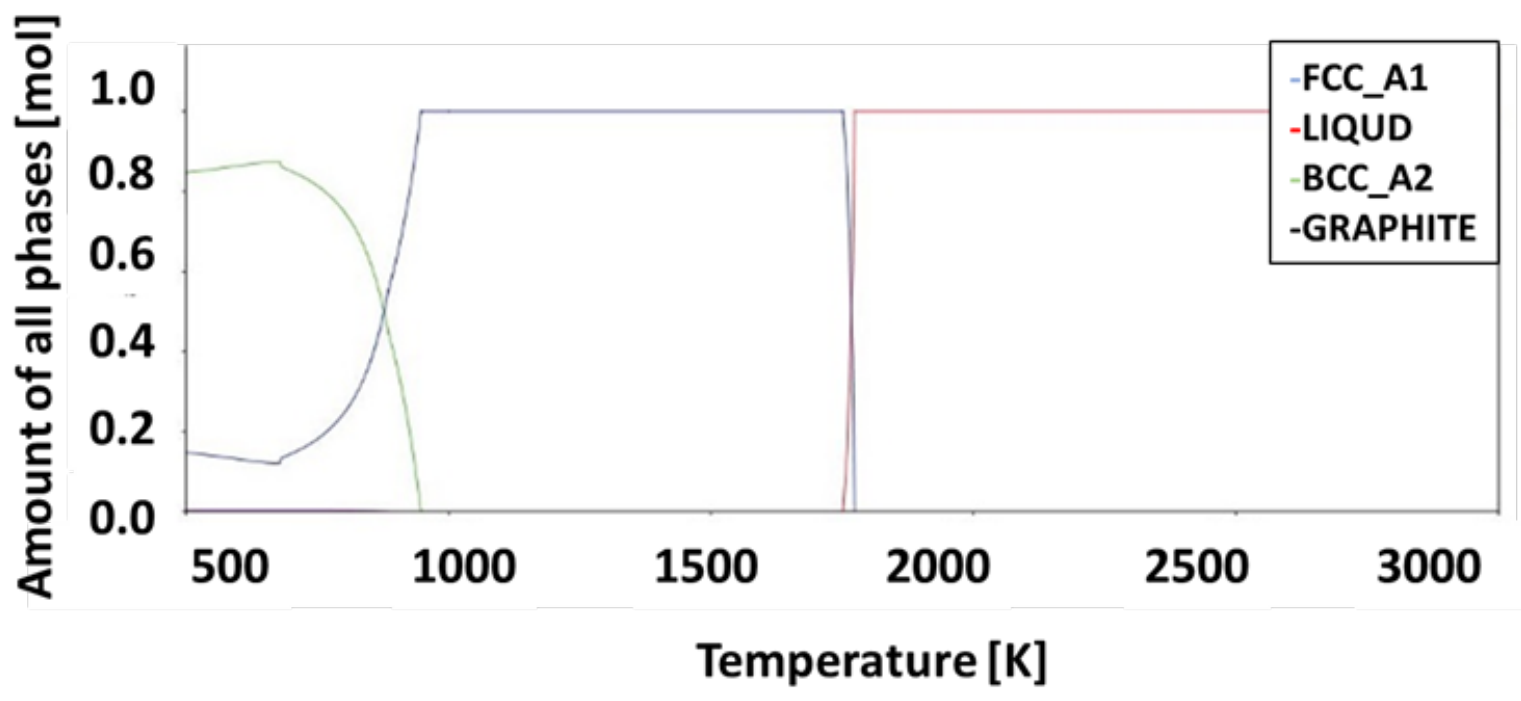

Fig. 2: Property diagram of Fe-10Ni-0.1C, the figure shows the number of phases that the material exhibit over a pre-assigned rangeof temperatures.

A precipitation module (PRISMA) integrated in Thermo-Calc $\AA$, is used to simulate concurrent nucleation, growth and coarsening of second phase in multi-component systems (see figure 3), some of the outputs of this module are:

- Particle size distribution (PSD).
- Number density.

- Average particle radius.

- Volume fraction.

- Average compositions.

- Nucleation rate.

- Critical radius.

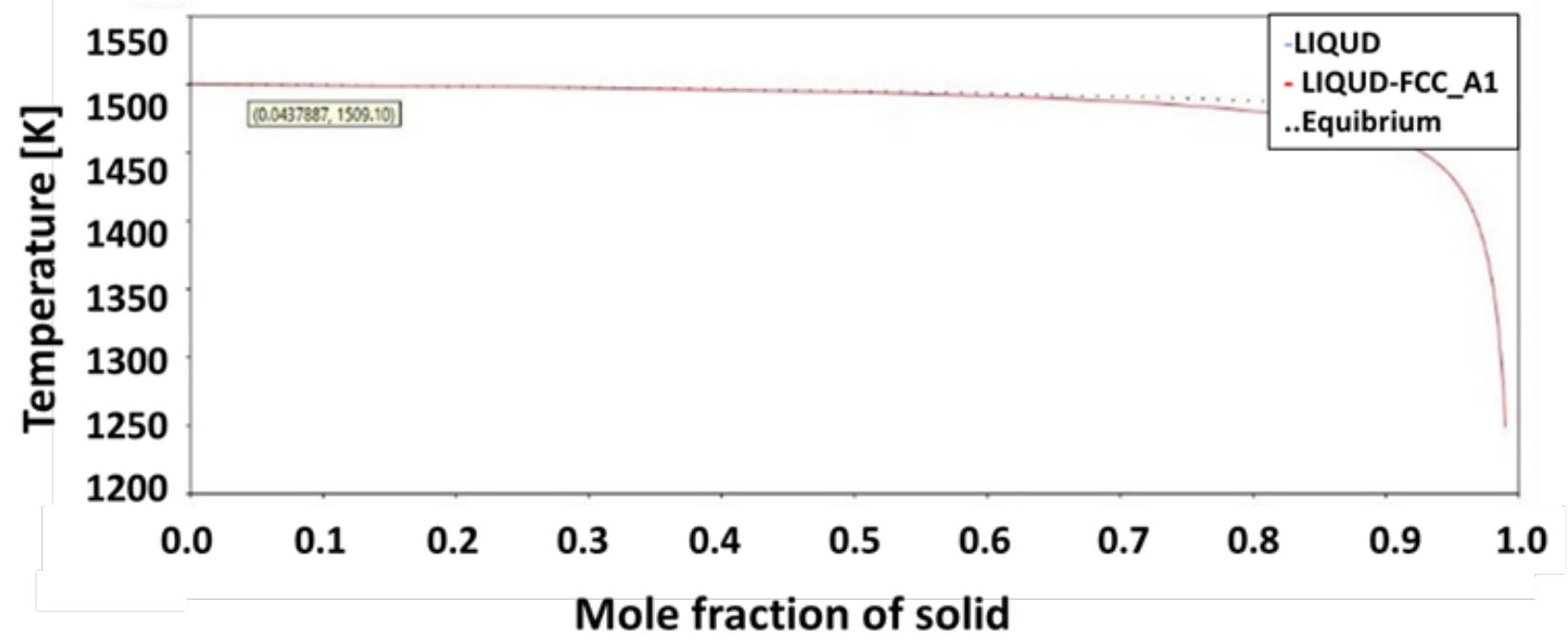

Fig. 3: Scheil Solidification simulation showing the effect of non-equilibrium solidification on Fe-10Ni-0.1C solid mole fraction, the equilibrium solidification is shown in dotted lines and the difference between both lines indicates the presence of micro-segregation.

Figure 4 shows the volume fraction and radius variation with time of $\mathrm{Fe}-\mathrm{C}$ precipitate (cementite) at $100{ }^{\circ} \mathrm{C}$ isothermal precipitation calculation. And, figure 5 shows the precipitation calculation of $\mathrm{Al} 3 \mathrm{Sc}$ in an Aluminum Scandium alloy at $350^{\circ} \mathrm{C}$ and shows size distribution variation at different time domains. 


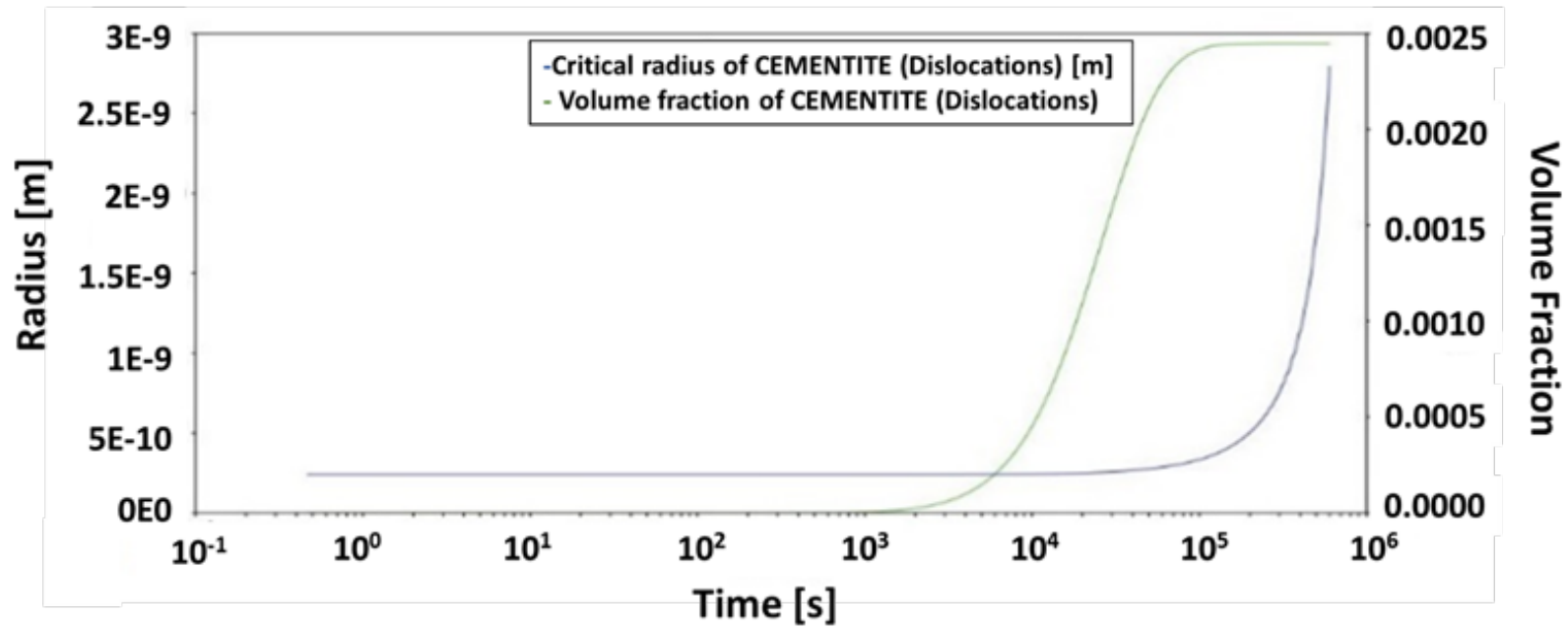

Fig. 4: Volume fraction and radius variation with time of $\mathrm{Fe}-\mathrm{C}$ precipitate (cementite) at $100{ }^{\circ} \mathrm{C}$ isothermal precipitation calculation.

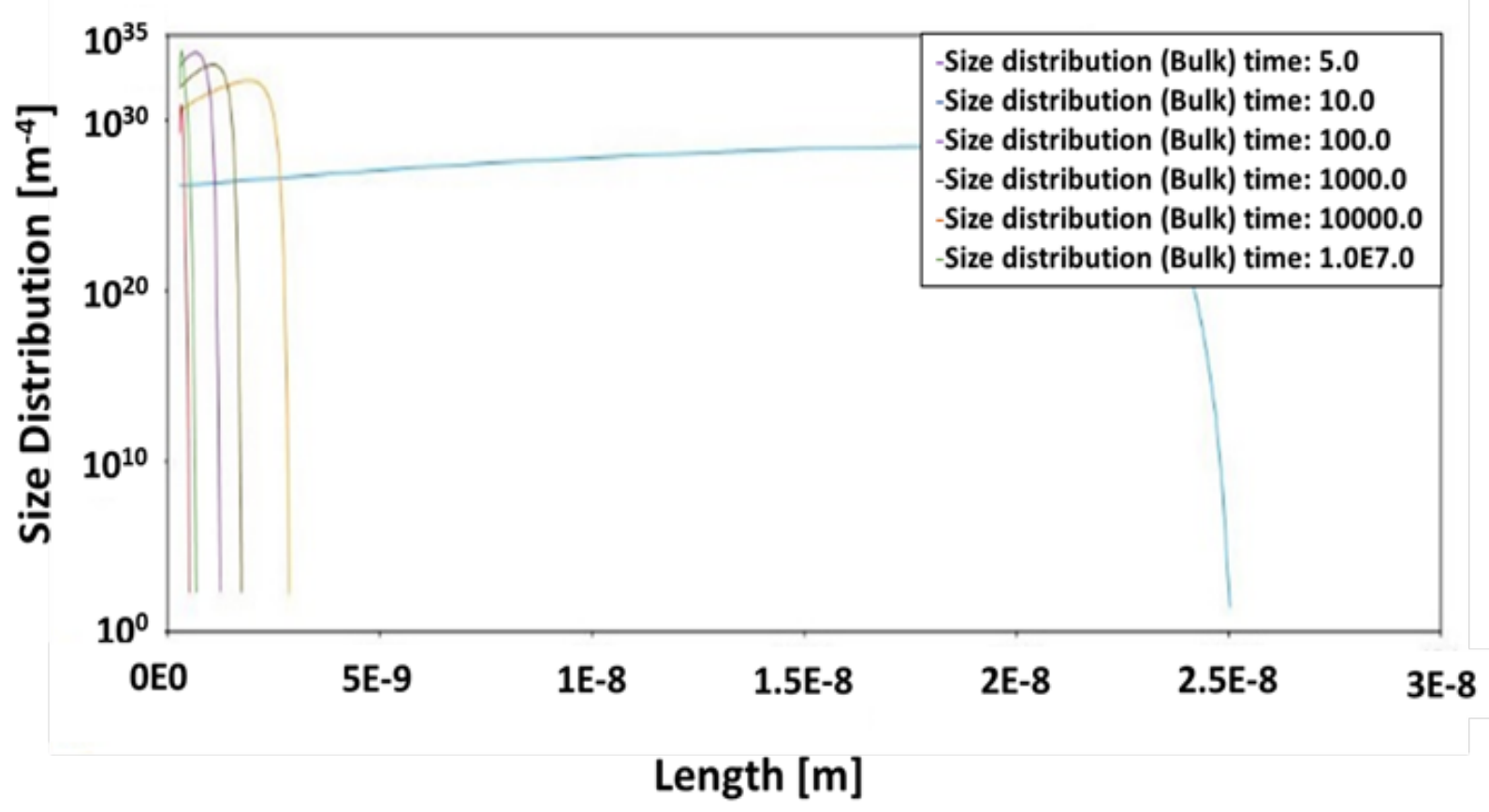

Fig. 5: Precipitation calculation of $\mathrm{Al3Sc}$ in an Aluminum Scandium alloy at $350^{\circ} \mathrm{C}$ showing size distribution variation at different time domains.

Another module integrated into Thermo-Calc ${ }^{\circledR}$, is the diffusion module (DICTRA). This module can simulate:

- Homogenization (see figure 6).

- Micro-segregation during solidification.
- Growth/dissolution of precipitates.

- Coarsening of precipitates.

For AM, the process is not isothermal so, a custom thermal profile as shown in figure 7 can be an input for Thermo-Calc $\AA$, DICTRA and PRISMA. 


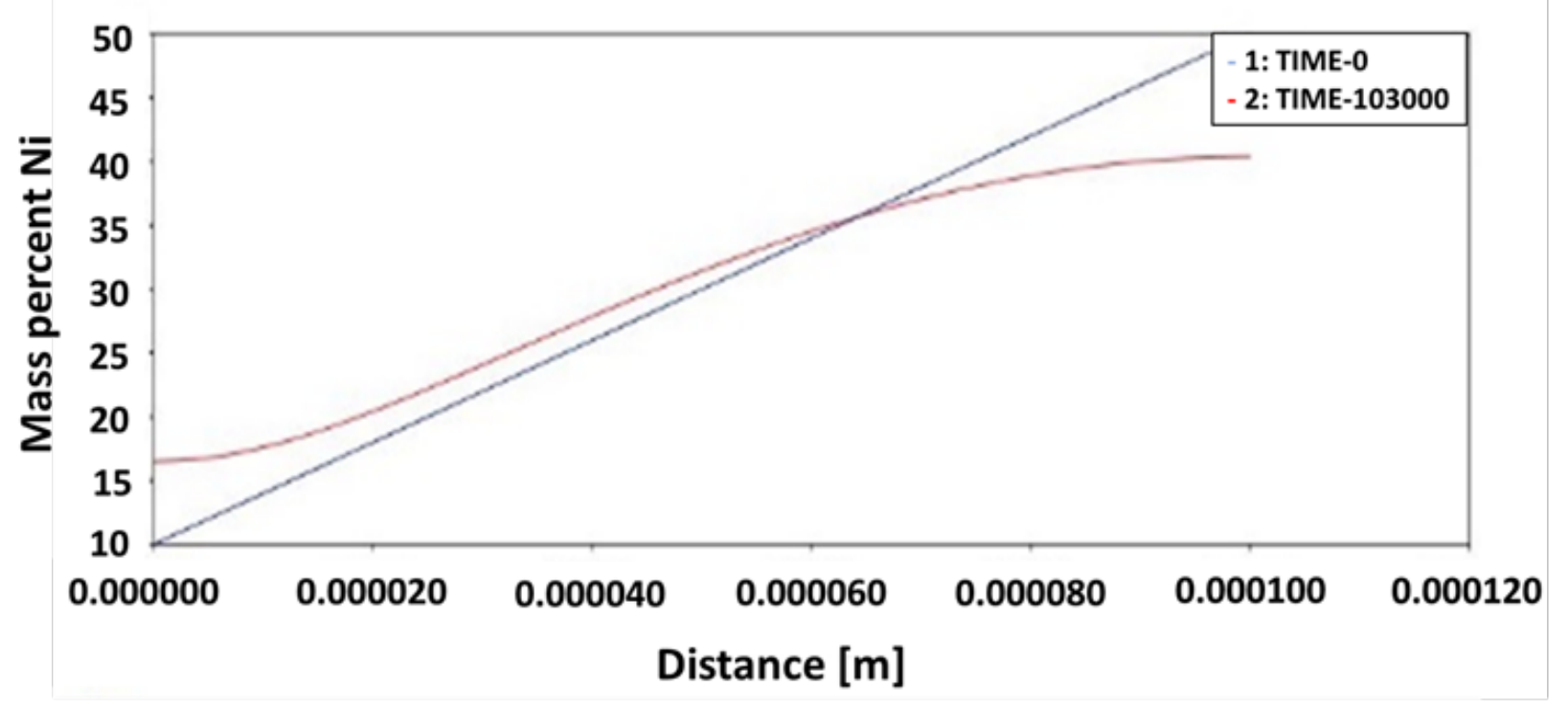

Fig. 6: Simulation of homogenization of Fe- $0.1 \mathrm{Ni}$ at an isothermal temperature of $1400 \mathrm{~K}$ in a planar domain.

\begin{tabular}{|l|l|}
\hline Tme [s] & Temperature $[$ K] \\
\hline 0.0 & 1640.0 \\
\hline 0.002 & 1000.0 \\
\hline 0.004 & 950.0 \\
\hline 0.005 & 900.0 \\
\hline 0.006 & 1700.0 \\
\hline 0.003 & 1050.0 \\
\hline 0.01 & 950.0 \\
\hline 0.012 & 1090.0 \\
\hline 0.013 & 980.0 \\
\hline & \\
& \\
& \\
& \\
& \\
& \\
& \\
& \\
& \\
& \\
& \\
& \\
&
\end{tabular}

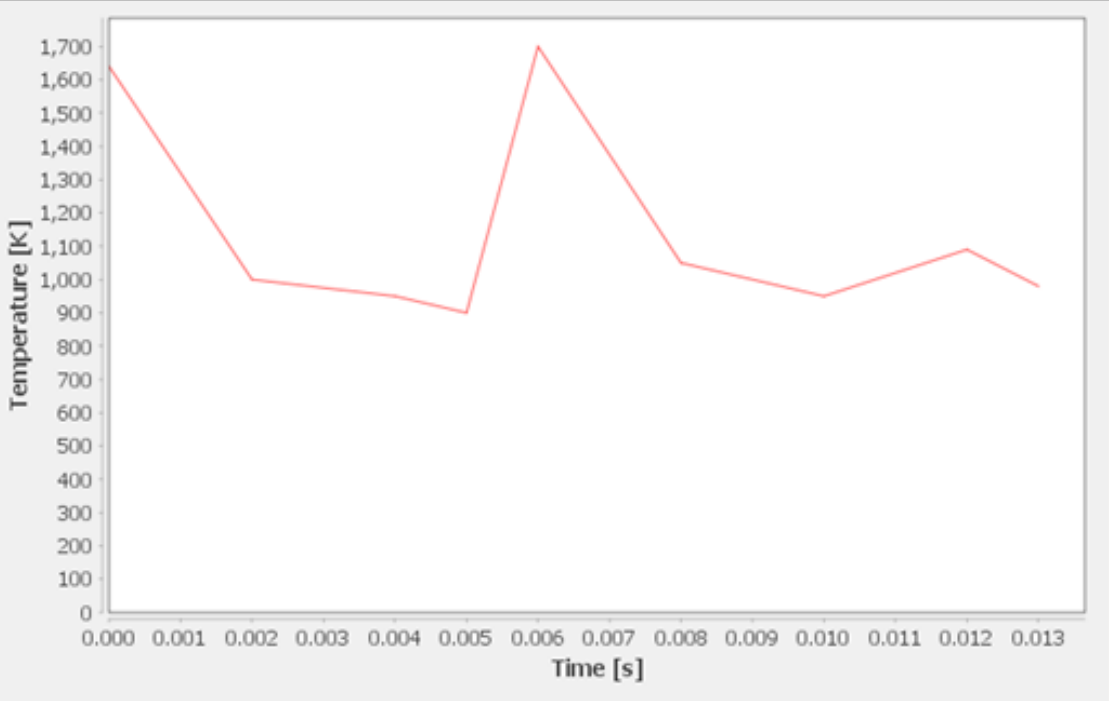

Fig. 7: Thermal profile of a laser beams with three runs over a substrate.

\section{B- MICROSCALE MODELLING}

Using the output of the CALPHAD software, the microstructure of the material can be predicted and generated. Generating microstructure for the whole mesoscale part and then simulating it would prove very computationally expensive. Therefore, a Representative Volume Element (RVE) that is large enough is selected to include all features of the materials and interactions of the microstructure. Then the Crystal Plasticity (CP) model is run after setting the boundary condition of the RVE. The CP Phenomenological based models areused/ integrated from DAMASK Spectral Solver. An isostrain homogenization scheme is used to ensure the homogeneity of the RVE.

In this work, the ordinary Voronoi tessellation method as a commonly accepted method to generate RVE microstructures is used ${ }^{[19-22]}$. 


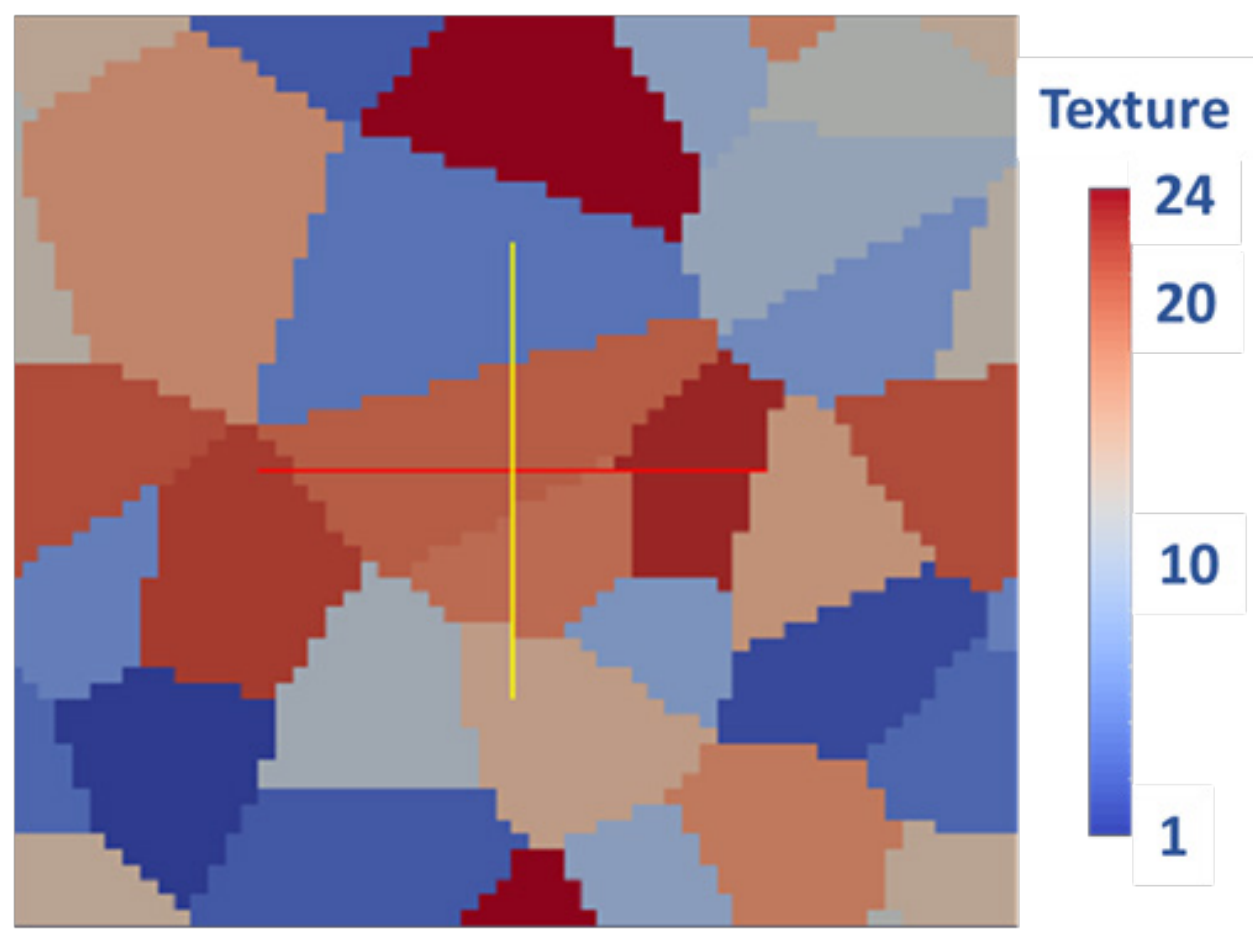

Fig. 8: The 24 grains microstructure generated using ordinary Voronoi tessellation method.

After generating the microstructure as shown in figure 8 , the boundary conditions are then set to constrain and apply necessary deformation. In the preliminary simulation results, the material was compressed along the $\mathrm{Y}$-axis and the lower edge was constrained. This work follows Hatem and Zikrymodels as presented ${ }^{[23-28]}$. Therefore, the Crystal plasticity (CP) phenomenologicalbased modelling along with specialized Fourier spectral solvers; that utilize fast Fourier transform (FFT), are used to study the material behaviour as well as the failure in the material. The following CP formula is utilized by ${ }^{[25-30]}$;

$$
\dot{\gamma}^{\alpha}=\dot{\gamma}_{0}\left|\frac{\tau^{\alpha}}{\tau_{\mathrm{c}}{ }^{\alpha}}\right|^{m} \operatorname{sgn}\left(\tau^{\alpha}\right)
$$

Where $\tau_{\mathrm{c}}^{\alpha}$ is the slip resistance, $\gamma 0$ and $\mathrm{m}$ are material parameters that quantify the reference shear rate and the sensitivity rate of slip, respectively.

The influence of any slip system $\beta$ on the hardening behavior of slip system $\alpha$ is given by:

$$
\tau_{c}{ }^{\alpha}=h_{\beta}\left|\dot{\gamma}^{\beta}\right|
$$

where $h_{\alpha \beta}$ is referred to as the hardening matrix which can be calculated as:

$$
\mathrm{h}_{\alpha \beta}=\mathrm{q}_{\alpha \beta}\left[\mathrm{h}_{\mathrm{o}}\left(1-\frac{\tau_{\mathrm{c}}{ }^{\beta}}{\tau_{\mathrm{s}}}\right)^{\mathrm{a}}\right]
$$

where, $h_{0}$, a and $\tau_{\mathrm{s}}$ are slip hardening parameters.

The resulting microstructure is modelled by $\mathrm{CP}$ based models to address the different failure mechanisms in the material. As the total plastic shear strain is the sum of the shear strain rate on each active slip system, this work results only the total plastic shear contour. After applying the uniaxial compression load along [01 $\overline{0}$ ], the total plastic shear strain contour (see figure 9) shows a shear strain localization along the [110]. The maximum value of the plastic shear strain is 0.337 that localized along the band direction of [110].

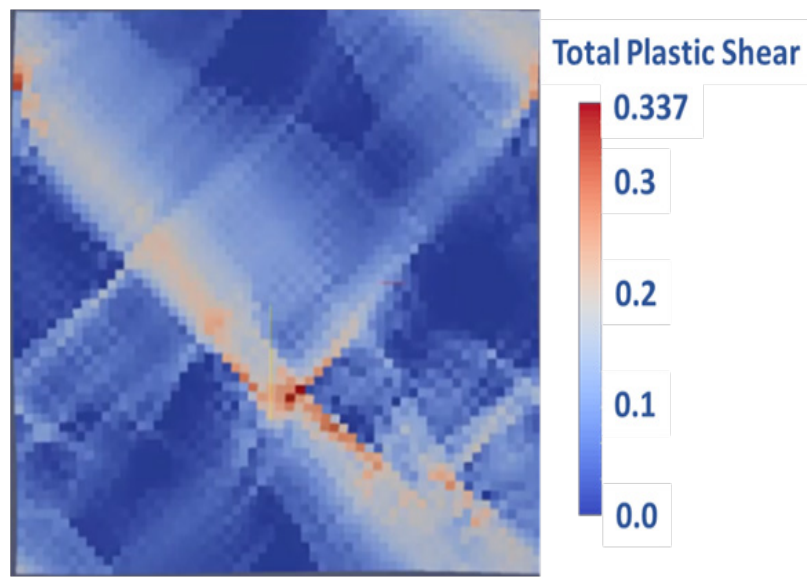

Fig. 9: Total Plastic Shear contour shows the shear strain localization in the material due to compression along $\mathrm{Y}$-axis $[0 \overline{10}]$. 


\section{C- MACROSCLAE MODELLING}

The last part of the procedure is to design the macroscale product (the hip implant). As shown in figure 10, a precise geometrical CAD design is accomplished using SolidWorks. Then it undergoes to finite element simulation to check whether it will withstand the load or not.

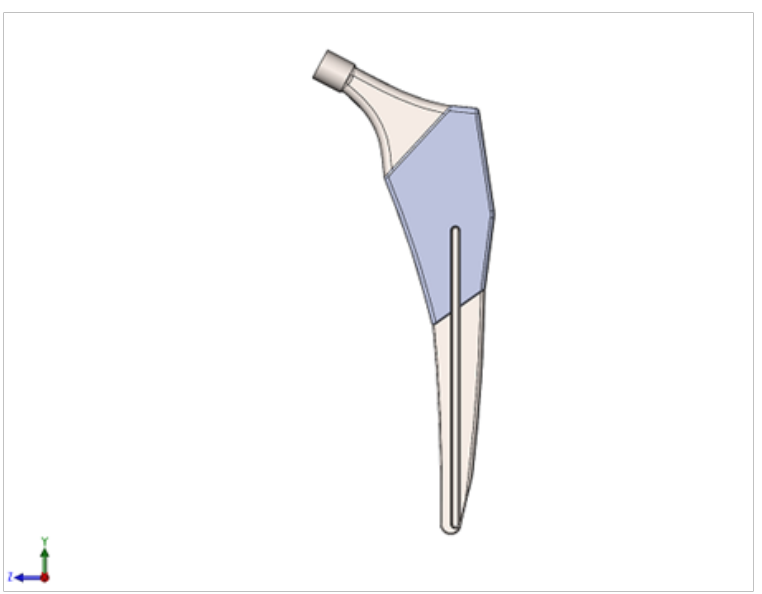

Fig. 10: Geometrical design (CAD)of the 3-D hip implant structure.

As illustrated in Figure 11, a Finite element analysis is done on the CAD model using ABAQUS software to simulate operating environment with the customdesigned implant. The aim of the model is to predict the in-operation stress state of the part and its behaviour with the surrounding parts, and the following is Von Mises analysis. The maximum value of Von Mises stress is 330 $\mathrm{GPa}$ whereas the minimum value is $0.45 \mathrm{MPa}$.

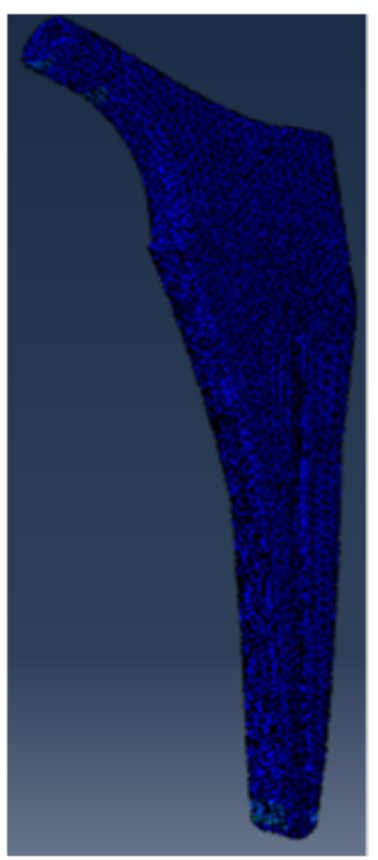

Von Mises Stress

\section{$3.3 e+10$}

$2.7 e+10$

$2.0 \mathrm{e}+10$

$1.5 e+10$

$1.2 \mathrm{e}+10$

$5.5 e+09$

$4.5 e+05$

Fig. 11: 3-D contour shows ABAQUS results of the Von-Mises Stressof the hip implant.
According to the fixation of the part, the displacement is extensive at the top of the part to reach 1.03 and it is fixed at the end with zero value; as it is shown in figure 12 .

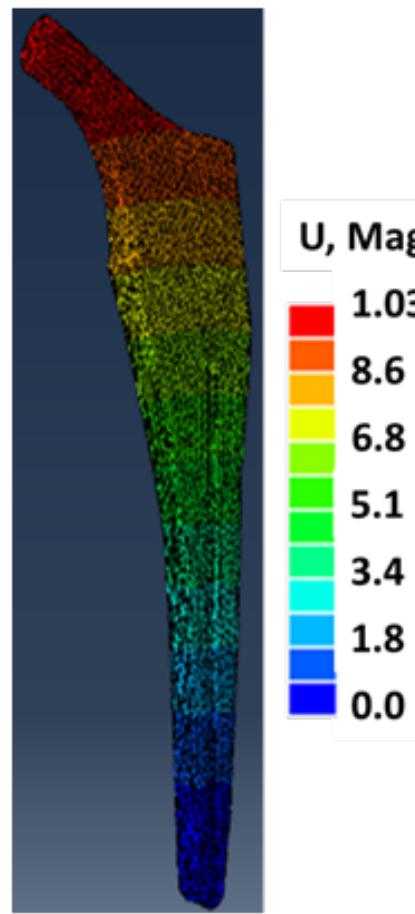

Fig. 12: 3-D contour shows ABAQUS results of the Displacement (U) along the stem.

\section{CONCLUSION}

To achieve optimal material integrity and outstanding functionalities, different processing parameters impact on AM process are explored. Furthermore, development of a design framework based on general multi-physics modelling is suggested. This research focuses on highstrength stainless steel, especially 17-4 PH stainless steel. The proposed framework includes:

(1) Coupled thermal/microstructural prediction of DMLS process.

(2) Simulating precisely microstructures of the complex high-strength stainless steel.

(3) Developing numerical models to be used for AM parts.

\section{REFERENCES}

[1] DebRoy, T., Wei, H. L., Zuback, J. S., Mukherjee, T., Elmer, J. W. Milewski, J. O., and Zhang, W. (2018). Additive manufacturing of metallic components-process, structure and properties. Progress in Materials Science, 92, 112-224.

[2] Zhang, B., Li, Y., and Bai, Q. (2017). Defect formation mechanisms in selective laser melting: a review. Chinese Journal of Mechanical Engineering, 30(3), 515-527.

[3] Lin, X., Cao, Y., Wu, X., Yang, H., Chen, J., and Huang, W. (2012). Microstructure and mechanical properties of laser forming repaired 174PH stainless steel. Materials Science and Engineering: A, 553, 80-88. [4] Rafi, H. K., Pal, D., Patil, N., Starr, T. L., and Stucker, B. E. (2014) Microstructure and mechanical behavior of 17-4 precipitation hardenable steel processed by selective laser melting. Journal of materials engineering and performance, 23(12), 4421-4428.

[5] Shalan, K.M., et al. Multiscale model and experimental study of damage in piezoelectric fiber-based composite. in EWSHM-7th European Workshop on Structural Health Monitoring. 2014. 
[6] El-Etriby, A.E., et al. A Multi-scale based model for composite materials with embedded PZT filaments for energy harvesting. in Proceedings of the TMS Middle East-Mediterranean Materials Congress on Energy and Infrastructure Systems (MEMA 2015). 2015. Springer.

[7] Khafagy, K.H., T.M. Hatem, and S.M. Bedair, Impact of embedded voids on thin-films with high thermal expansion coefficients mismatch. Applied Physics Letters, 2018. 112(4): p. 042109.

[8] Khafagy, K.H., Hatem, T.M. and Bedair, S.M., Modelling of III-Nitride Epitaxial Layers Grown on Silicon Substrates with Low DislocationDensities. MRS Advances. 2019;4(13):755-60.

[9] El-Etriby, A.E., et al. A multiscale-based approach for composite materials with embedded PZT filaments for energy harvesting. in SPIE Smart Structures and Materials + Nondestructive Evaluation and Health Monitoring. 2014. SPIE.

[10] Wagih, M., et al., Discerning enhanced dislocation plasticity in hydrogen-charged $\alpha$-iron nano-crystals. Materials Research Letters, 2015. 3(4): p. 184-189.

[11] Shi, J., M. Zikry, and T.M. Hatem, Inelastic behavior and grainboundary effects in polycrystalline materials. MRS Online Proceedings Library Archive, 2006. 978.

[12] Hendy, M.A., Hatem, T.M. and El-Awady, J.A., 2018. Atomistic Simulations of Carbon and Hydrogen Diffusion and Segregation in Alfa-Iron Deviant CSL Grain Boundaries. MRS Advances, 3(45-46), pp.2795-2800.

[13] Kaufman, L. and J. Ågren, CALPHAD, first and second generation Birth of the materials genome. ScriptaMaterialia, 2014. 70: p. 3-6.

[14] Shanthraj, P., T.M. Hatem, and M.A. Zikry, Microstructural Modeling of Failure Modes in Martensitic Steel Alloys. MRS Proceedings, 2011. 1296: p. mrsf10-1296-o05-10.

[15] Horstemeyer, M., Multiscale Modeling: A Review. 2009. 87-135.

[16] Yip, S., Handbook of Materials Modeling. 2005, Dordrecht: Springer.

[17] E., W., Principles of Multiscale Modeling. 2011: Cambridge University Press.

[18] Steinhauser, M., Computational Multiscale Modeling of Fluids and Solids : Theory and Applications. Vol. 2nd ed. 2017, Berlin: Springer.

[19] Jafari, R. and M. Kazeminezhad, Microstructure generation of severely deformed materials using Voronoi diagram in Laguerre geometry: Full algorithm. Computational Materials Science, 2011. 50(9): p. 2698-2705.
[20] Kalidindi, S.R. and L. Anand, An approximate procedure for predicting the evolution of crystallographic texture in bulk deformation processing of fcc metals. International Journal of Mechanical Sciences, 1992. 34(4): p. 309-329

[21] Wu, X., et al., Prediction of crystallographic texture evolution and anisotropic stress-strain curves during large plastic strains in high purity $\alpha$-titanium using a Taylor-type crystal plasticity model. Acta Materialia, 2007. 55(2): p. 423-432.

[22] Salem, A.A., S.R. Kalidindi, and S.L. Semiatin, Strain hardening due to deformation twinning in $\alpha$-titanium: Constitutive relations and crystalplasticity modeling. Acta Materialia, 2005. 53(12): p. 3495-3502.

[23] Hamza, M.H., Hendy, M.A., Hatem, T.M. and El-Awady, J.A., 2018. Impact of angular deviation from coincidence site lattice grain boundaries on hydrogen segregation and diffusion in $\alpha$-iron. MRS Communications, 8(3), pp.1197-1203.

[24] Hatem, T. and M. Zikry, A model for determining initial dislocationdensities associated with martensitic transformations. Materials Science and Technology, 2011. 27(10): p. 1570-1573.

[25] Hatem, T. and M. Zikry, Deformation and Failure of Single-Packets in Martensitic Steels. Computers, Materials, and Continua, 2010. 17(2): p. 127-147.

[26] Hatem, T. and M. Zikry, Dislocation density crystalline plasticity modeling of lath martensitic microstructures in steel alloys. Philosophical Magazine, 2009. 89(33): p. 3087-3109.

[27] Hatem, T.M. and M.A. Zikry, Modeling of Lath Martensitic Microstructures and Failure Evolution in Steel Alloys. Journa of Engineering Materials and Technology, 2009. 131(4) p. 041207-041207-10.

[28] Hatem, T. and M. Zikry, Shear pipe effects and dynamic shear-strain localization in martensitic steels. Acta Materialia 2009. 57(15): p. 4558-4567.

[29] Roters, F., Eisenlohr, P., Hantcherli, L., Tjahjanto, D.D., Bieler, T.R. and Raabe, D., 2010. Overview of constitutive laws, kinematics, homogenization and multiscale methods in crystal plasticity finite-element modeling: Theory, experiments, applications. Acta Materialia, 58(4), pp.1152-1211.

[30] Asaro, R.J. and Needleman, A., 1985. Overview no. 42 texture development and strain hardening in rate dependent polycrystals. Acta Metallurgica, 33(6), pp.923-953. 